\title{
Soft exosuit-a review
}

\begin{abstract}
Exosuit is nothing but a device or robot which works in different areas, such as - military, medical or lifting some load. Generally, it is not fully covered like a shield because it is made by using cables motors and some useful mechanism. Exosuit is broadly developed for both rehabilitation and to augment user's physical performance. In this paper, we will discuss the design considerations, different types of actuator, or actuation processes, sensors and some particular mechanisms and also define some limitations of the same. We used a new approach with respect to the current scenario in this paper. Here, we are only studying about the relatable importance point which is directly connected to exosuit. There are many reasons for low employment of exosuit in the industry with new technology in the automotive and logistics. At this time there are very few exoskeletons available to purchase as a final and certified product on the free market. Generally, exosuit is a worn mechanical device that works parallel with the user; it has the potential to enhance the requirements of a human. The exosuit utilizes electric or hydraulic actuator support or augments human muscle strength which helps in transporting a heavyweight object.
\end{abstract}

Keywords: exosuit, actuator, human muscle, industry, technology
Volume 6 Issue 2 - 2020

\section{Aman Jain, Kalpit Jain}

Department of Mechanical Engineering, Poornima College of Engineering, India

Correspondence: Aman Jain, B.Tech Scholar, Department of Mechanical Engineering, Poornima College of Engineering, Jaipur, India, Email2016pcemeaman0I0@poornima.org

Received: April 20, 2020 | Published: June 30, 2020

\section{Introduction}

Exosuit is a wearable suit which is used in the different areas nowadays. In recent decades, researches have been done, across the globe to improve this technology. This result is to improve practical knowledge. The exosuit with an improved technology in the field of mechanical design, electronics engineering, biomedical design and in computerized reasoning plays a vital role in the field of rehabilitation for enhancing human performance. ${ }^{1}$ The concept was first mentioned in 1966. Current area of research includes in exosuit is an upper limb, lower limb hand movements and many more. According to a new research report of the global market, the cost of the exoskeleton is USD 765 thousand to USD 3900 thousand from 2019 to 2025. Generally, a number of exosuit have been developed for tasks such as heavy lifting it which can extend physical therapy to the home and improve outcomes for stroke patients. Since 2013, improvements in the actuator and sensors have been seen and these systems have become portable, also for commercial application - in reducing the weight and power requirement and to this end, some groups have proposed a quasi-passive architecture in an effect to reduce the exoskeleton's energy consumption. ${ }^{2}$

Further use and development were found in the military sector to enhance soldier's strength. In medical field, exosuit was developed to support disabled and to handicapped people. Current research is working on its design adding new sensors to measure human kinematics and the suit- human interaction force that are a robust complaint and cost effective. At this time there are very few exosuit available to purchase as a final certified product on the free market. Technical definition standardization and certification or rather a declaration of conformity are still not found or clearly solved the challenges for the most of the world markets. Currently, there are no Exosuit commercially available which are suitable for a generic workplace requirement. ${ }^{3}$ The major issue for robot design across all targets is achieving human-robot interaction [HRI]. The first challenge is to ensure freedom to motion and adaptation to different morphologies and both genders. The second challenge is to affect the exchange force between the user and exosuit. ${ }^{4}$ In response to a musculoskeletal complaint related to hand tool use and material handling, various intervention have been introduced into the occupational environment including cranes and other lift assist device, among many advantages, these devices also have significant cost but reduced flexibility which can prove to be both time and space consuming. Currently, the exosuit can be divided in two ways.

\section{a. According to their power and}

b. According to the structure

Power type again divided into two parts

i. Active: which requires power

ii. Passive: which has no external force

Structure types are also divided into two parts

i. Hard structure and

ii. Soft structure

\section{Actuator}

Different types of actuator are considered with respect to the anatomical joint for the human body. An independent actuator can provide one degree of freedom but shoulder and some joint need multiple degrees of freedom than it is select on the basis of requirement. Generally, actuators are classified into three types

i. Active actuators,

ii. Semi-active actuators and

iii. Passive actuators.

The active actuator can produce a variable range of motion with different speed and torque. The semi-active actuator is a special type of actuator which cannot produce any active force in the joint but impose resistive force. The passive actuator provides a supporting force to the joint it is based which use their elastic property to generate force without using the source of energy. Also, some new type of actuator is found in the industry such as artificial muscle, 
Shape Memory Alloy(SMA), Electro-Active Polymer(EAP). ${ }^{5}$ Some custom actuators were made to minimize weight for the size and force requirement based on the biological specifications. Air or we can say the compressed air is used to or supplied to the actuator for expanding and shorting the length for providing actuation. Another interesting hardware approach is to use a Series Elastic Actuator (SEA). A SEA is essential a spring element placed in series with the stiff actuator to provide passive compliance. ${ }^{6}$ Mckibben actuator has been suggested as an actuator whose performance is similar to biological muscle. This is true for the force-length relationship, but little is known regarding the force-velocity relationship for applying a torque to a biological joint, these actuator require a means to apply a reaction force to another part of the human body and this force can be quite high by this thing skin adhesive were used to maintain the position and these causes discomfort. If the duration of actuation is adjusted to zero milliseconds then they generated zero force. ${ }^{2}$ Now, most of the exosuit use an electric motor for actuation.

\section{Design}

Some important design considerations are - lightweight, minimal inertia, non-restrictive, comfort during operation, easy movement and the basic or important are actuator type, the degree of freedom, control strategy, applied joint, application domain, Power transmission method, human intention detector and design concept. Other desirable features include small size and weight of the exosuit the use of breathable material to prevent sweating and a design that allow the user to easily put on and remove the exosuit without external force. In the soft exoskeleton, if we are using the aluminium, steel and composite material for rigid links for the functional mechanical action like straps and bets. The major drawback of rigid element in that this produces large inertia and misalignment which can lead to unnatural motion and discomfort also extreme injuries. ${ }^{7}$ Electro-Active Polymer (EAP) newly developed an electrical material advantage of this is high bandwidth and a higher level of electrical to mechanical power conversion ratio. Generally, nowadays some soft actuators and 3D printed gloves are developed for lightweight but one of the main problems of using soft actuator is in the mathematical modeling which is highly complex because of their nonlinear behavior. Power augmentation and rehabilitation wearable robot must be

i. Inherently safe because they are in direct contact of a human.

ii. Lightweight for easy use and portability.

iii. Able to lift a person without extensive adjustment and calibration. ${ }^{1}$

With the upgraded in material and design now the active, component are added through a cable attached to the motor and the cable inject energy during a specific time of the gait via controllers that use sensors to monitor the wearer's movement.

\section{Mechanism}

Generally, the compressed air is supplied to actuators for expanding and shorting the length for providing actuation when the input pressure is changed force is automatically changed and the air or compressed air was supplied from the compressor to the system uses of tubing. There are many limitations in the pneumatic system which is limited stroke length and difficult to achieving accurate force control for overcoming this limitation we have to introduce cable based actuator in which motor away from the joint and more convenient. ${ }^{7}$ The exosuit forms a kinematic change in close contact with the human body and may provide support rigidity protection or augmentation of sensitivity. They can be active or passive.

\section{i. Active: they provide external strength \\ ii. Passive: they provide support and protection}

Generally, power should be transmitted through the cable based, gear based, linkage based or combination of two or more power transmission method (Hybrid based). Gears are used to reduce the speed which increases the weight and reduce the efficiency typically from $70 \%$ to $50 \%{ }^{5}$ Brushless DC motor can provide better power to weight ratio. A hand exoskeleton was also introduced in the industry which was work on a signal that the user can interact directly with the nervous system electrical brain activity is translated into control signal via Brain-Computer Interface(BCI) and then sent the hand exoskeleton. The mechanism and control can be evaluated with a rigid body simulation program or general purpose simulation software such as Adams, Matlab and Modelica.

\section{Sensors}

Sensors are used to capture information or intention from human to be sent to the exosuit controller. Generally, sensors are categorized into two main groups, Cognitive sensors and physical based sensors.

i. Cognitive sensors: it reads intention by measuring the electric signal from the nervous system (EEG, MSS, EMG).

ii. Physical-based sensors: it reads intention by measuring the motion of human limbs using position, force, pressure.

\section{Limitations}

Brain-Computer Interface (BCI) also has some limitations that these cannot be justified or differentiated between the various types of objects and the type of grip that should be applied. A simulation architecture was developed that was the musculoskeletal model from Open Sim but the limitations of Open Sim concept of combined simulation is that this works for constant velocity and the transition occurring between a limited set of torque magnitude. Electro-Active Polymer (EAP) the only disadvantage is very low torque weight ratio only this is the reason this is not fit for exosuit actuation. ${ }^{5}$

\section{Conclusion}

According to the current research, the cost of the exosuit is very high and the different mechanisms are used nowadays to enhance the working principles and efficiency. But the cable based power transmission is more effective as compared to the pneumatic control because of pneumatic exosuit is heavy and not easy to portable type. Many actuators are also designed for giving better efficiency. The 3D printed gloves are more useful for hands because of the lightweight. Sensors play an important role in increasing the level of understanding between the human and exosuit at the time of performing the operation. The only thing at the end is that exosuit will be more beneficial in the future in the field of military service, biomedical for enhancing the ability of patients and also in daily life for reducing the efforts of a normal person.

\section{Funding}

None. 


\section{Acknowledgments}

None.

\section{Conflicts of interest}

The authors declare that there was no conflict of interest.

\section{References}

1. Hassanin Al-Fahaam, Steve Davis, Samia Nefti M. The design and mathematical modelling of novel extensor bending pneumatic artificial muscles (ebpams) for soft exoskeletons. Robotics and Autonomous Systems. 2018;99:63-74.

2. Michael Wehner, Brendan Quinlivan, Patick M Aubin. A Lightweight Soft Exosuit for Gait Assistance. IEEE International Conference on robotics and Automation. 2013.

3. Christian Dahmen, Frank Wölleckea, Carmen Constantinescub. Challenges and possible solutions for enhancing the workplaces of the future by integrating smart and adaptive exoskeletons. Procedia CIRP. 2018;67:268-273.
4. Carine Rognon, Stefano Mintchev, Fabio Dell'Agnola, et al. Fly jacket: An Upper Body Soft Exoskeleton for Immersive Drone Control. IEEE Robotics and Automation Letters. 2018;8(3):2362-2369.

5. Soumya KM, Venketesh ND. Comparative study of actuation systems for portable upper limb exoskeletons. Medical Engineering and Physics. 2018;60:1-13.

6. Caldwell DG, Tsagarakis NG, Sophia K, et al. "Soft" Exoskeletons for Upper and Lower Body Rehabilition- Design, Control and Testing. International Journal of Humanoid Robotics. 2007;4(3):549-573.

7. Leonardo Cappello, Dinh Khanh Binh, Shih Cheng Yen, et al. Design and Preliminary Characterization of a Soft Wearable Exoskeleton for Upper Limb. IEEE RAS/EMBS International Conference on Biomedical Robotics and Biomechatronics. 2016. 